national guidelines. Data analysis was performed using Stata v13.

Results A total of $712 \mathrm{MSM} / \mathrm{TGW}$ attended a VICITS clinic (91.4\% MSM, 8.6\% TGW) with 545 in Managua (484 MSM, $61 \mathrm{TGW}$ ) and $167 \mathrm{MSM}$ in Granada. Median age was 21 yo, median age at first sexual intercourse was 15 yo in both cities; $94.6 \%$ of MSM in Granada and $71.8 \%$ of MSM/TGW in Managua reported receptive anal sex in the last 30 days. Granada reported highest alcohol use in the last month (75.5\%). Condom use in the last sex was reported by $71.3 \%$ MSM/TGW in Managua and $93.8 \%$ MSM in Granada. HIV prevalence was $4.9 \%$ in Managua and $0.6 \%$ in Granada. Syphilis prevalence was $4.6 \%$ Managua and $0.6 \%$ in Granada.

Conclusion Managua showed higher prevalence of HIV and Syphilis and sexual risk behaviours in MSM/TGW than Granada. Our results show young people getting infected in both cities, underscoring the need to implement additional efforts to control HIV epidemic among this young key population in Managua and Granada.

Disclosure of interest statement We declare that we have no conflicts of interest.

\section{P09.40 PREVALENCE OF CURABLE SEXUALLY TRANSMITTED INFECTIONS IN PREGNANT WOMEN IN LOW- AND MIDDLE-INCOME COUNTRIES FROM 2010-2015: A SYSTEMATIC REVIEW}

HI Shull*, JD Klausner. David Geffen School of Medicine at UCLA, Los Angeles, California, USA

\section{$10.1136 /$ sextrans-2015-052270.424}

Introduction Globally nearly 17,000 children under 5 years of age die each day. Preterm delivery is the number one cause of under-5 year mortality. Curable sexually transmitted infections (STIs) in pregnant women, specifically syphilis, Neisseria gonorrhoeae, Chlamydia trachomatis, and Trichomonas vaginalis, have been shown to cause preterm delivery through premature rupture of membranes, preterm labour, chorioamnionitis and congenital infection. There is a strong likely causal association between antenatal STIs and preterm delivery. Treating curable STIs may decrease under- 5 year mortality. Our objective was to conduct a systematic review and summarise curable STI prevalence estimates among pregnant women in low- and middleincome countries.

Methods PubMed was searched for studies reporting prevalence statistics for syphilis, Neisseria gonorrhoeae, Chlamydia trachomatis, and Trichomonas vaginalis infections among pregnant women in low- and middle-income countries. Studies published between 1/1/2010-3/1/2015 were included. Abstracts of all search results and the full text of all potentially eligible articles were reviewed.

Results 376 potentially relevant reports were identified. 76 studies met inclusion criteria, providing 116 point prevalence estimates for curable STIs in pregnant women, including a total of $3,594,777$ women. The median value (range) of the estimates for each STI were as follows: syphilis, 1.9\% (0-41.7\%);
N. gonorrhoeae, $1.6 \%(0-19.0 \%)$; C. trachomatis, 9.8\% (0.1$41.3 \%)$; and T. vaginalis, $11.2 \%$ (1.0-32.3\%). The median prevalence value of any STI was found to be $23.9 \%$ (10.3-33.7\%).

Conclusion Prevalence rates of curable STIs in pregnant women in low- and middle-income countries range from low to high and vary by country. Median prevalence values, however, are high and suggest a large population-level burden of untreated curable infections in pregnant women. Interventions to screen and treat pregnant women in low- and middle-income countries to reduce preterm delivery and subsequent under-5 years of age mortality need urgent evaluation.

Disclosure of interest statement We have no disclosures to make.

\section{P09.41 CHLAMYDIA TRACHOMATIS SCREENING AMONG PRETERM BIRTHS IN BRAZIL: AN EXPERIENCE FROM A UNIVERSITY HOSPITAL}

${ }^{1} \mathrm{R}$ Schmidt, ${ }^{1}$ RR Muniz, ${ }^{1} \mathrm{E}$ Cola, ${ }^{2} \mathrm{D}$ Stauffert, ${ }^{2} \mathrm{MF}$ Silveira, ${ }^{1} \mathrm{AE}$ Miranda*. ${ }^{1}$ Post-Graduation Program in Infectious Diseases, Universidade Federal Do Espirito Santo; ${ }^{2}$ Post-Graduation Program in Epidemiology, Universidade Federal de Pelotas

\subsection{6/sextrans-2015-052270.425}

Background Premature birth (PPT) is a major determinant of neonatal morbimortality with adverse consequences for health. The causes are multifactorial, with intrauterine infection probably explains most of these outcomes. It is believed that infection with Chlamydia trachomatis (CT) is also involved in PPT and premature rupture of membranes. Our goal was to study the prevalence of CT in pregnant women and associated factors related to cases of PPT attended in an University Hospital in Vitoria, Brazil.

Methods A cross-sectional study performed among parturient who have preterm birth in a University Hospital from June 2012 to August 2013. Participants answered a questionnaire including demographic, behavioural, and clinical data. A sample of urine was collected and screened for CT using polymerase chain reaction.

Results The prevalence of PPT in the hospital during the period of the study was $26 \%$. A total of 378 cases of PPT were registered, among them 323 women participated and were tested for CT, forty-five (13.9\%) had a positive result. $31.6 \%$ was up to 24 years old and women infected by CT were younger than the others $(\mathrm{p}=0.022)$. A total of $76.2 \%$ were married/living together, and CT was more frequent among the single ones $(\mathrm{p}=$ $0.018) ; 16.7 \%$ of women had their first sexual activity under 14 years old. The causes of prematurity were maternal-fetal in $40.9 \%$, rupture of the membranes in $29.7 \%$ and premature labour in $29.4 \%$. In multivariate analysis, being married was a protective factor for infection [OR $=12: 48$ (95\% CI: $0.24-$ 0.97)]. None of the other characteristics were associated with CT infection.

Conclusions This study shows a high prevalence of preterm birth and CT infection among parturient who have preterm birth. This high prevalence increases the need for defining screening strategies and assistance during the prenatal period.

Disclosure of interest statement There is no conflict of interest 